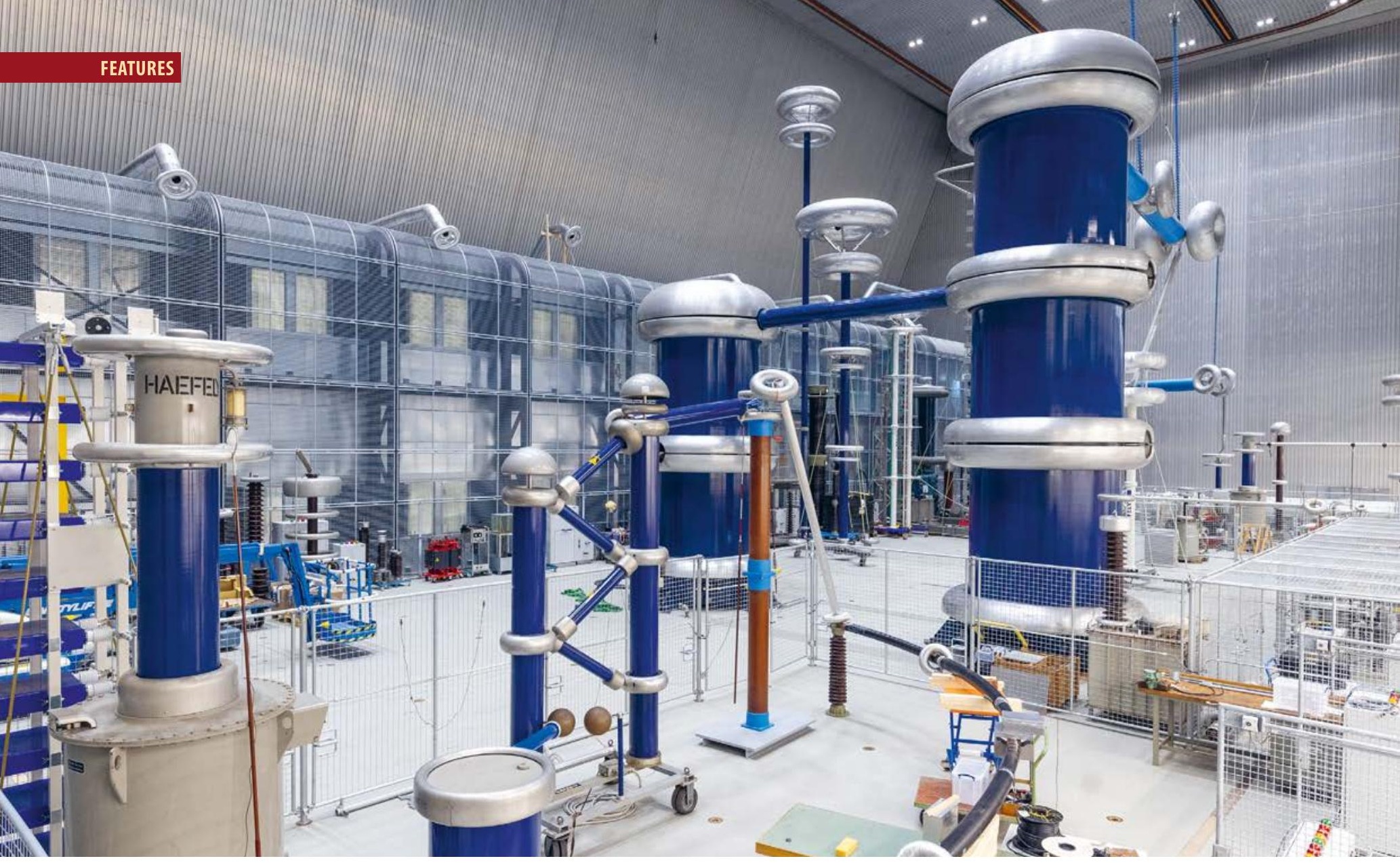

\title{
DEVELOPING THE FUTURE ELECTRICITY GRID
}

. Miro Zeman - Delft University of Technology, The Netherlands - DOI: https://doi.org/10.1051/epn/2021505

We are used to the continuous supply of electricity from a socket. Behind the socket lies a complex system of large power stations, high-voltage cables, transformers and a distribution network. Little has changed in the system over the last fifty years. The ambition to generate sustainable electricity from variable solar and wind energy has an immense impact on the electricity sector and requires major changes in our electricity grid and its operation.

$\Delta$ The Electrical Sustainable Power Lab

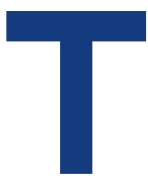
o facilitate the research needed for the transition of the power grid, Delft University of Technology in the Netherlands, recently has opened the new Electrical Sustainable Power Lab - the ESP lab. In the lab, the Dutch electricity grid is being prepared for the future. It is a lab that provides the ground for realistic tests and large-scale simulations using a digital twin of the real power grid. Using the knowledge and facilities of the ESP Lab, we aim to support and accelerate the energy transition. Let's see what is at stake.
Hybrid networks

Most existing electricity networks are based on alternating current (AC), while the electricity generated from sun and wind provides direct current (DC). To connect the direct current from sun and wind to the AC electricity grid new electronic components - inverters - are being developed. Inverters are very fast switches made of semiconductor materials. Unlike transformers, on which today's AC networks are based, inverters can convert a DC voltage to an AC voltage. To comply with the ambition to generate a large fraction of the electricity demand using 
sun and wind energy, the current electricity grids will have to be converted into hybrid AC/DC networks with a significant number of inverters. This is a major challenge.

\section{Variable energy sources}

Another challenge is the variability of sun and wind energy sources which, unlike the current fossil or nuclear energy sources, are not fully to our control. Sometimes the wind blows too hard and sometimes not at all. With the sun it is even more complicated: in addition to the day-night cycle, solar radiation can also be disturbed by clouds and there is a strong seasonal dependence in the amount and strength of the solar radiation. The variable nature of these energy sources has consequences for the operation of the electricity system. The operation of the current electricity systems with stable and controllable energy sources is based on the concept that the supply follows the demand. This is relatively easy to do, since the power requirements of consumers are very predictable and you can control the electricity production in the large power stations. In this way, supply and demand can easily be kept in balance.

However, this is not possible with sun and wind as electricity sources, since we cannot influence their availability. The electricity production of a solar plant can quickly switch between a lot of electricity and no electricity at all, depending on the weather. The inertia of the spinning heavy rotor of an electrical generator in traditional power plants is in stark contrast to the rapid response of solar panels to generate electricity. The latter can cause rapid fluctuations in the energy supply that can endanger the stability of the entire electricity grid. The problem can be solved if indeed the use of electricity would follow production or, if this is not possible, if electricity can be stored in, for example, batteries, which can provide the electricity quickly to balance supply and demand on the grid. Once they can be produced cheaply enough, they are expected to become one of the main suppliers of electricity during times when the sun is not shining and the wind is not blowing. Another, quickly emerging option is storage of excess electricity in green hydrogen that can solve seasonal differences in the availability of solar and wind energy.

\section{Stable grids}

The electricity grid is a huge system and there is always a chance on a disruption somewhere in the system. There are many possible causes for this, ranging from a broken coffee machine or a cable that is hit by excavation work or a fallen tree. Our current robust electricity grid can cope well with these disruptions thanks to the slowness and controllability of electricity production in the few large power plants. With electricity from renewable sources, supplied by countless wind turbines, photovoltaic systems and inverters, the disturbances are not easily absorbed and can have very devastating consequences for the electricity grid. Consequently, maintaining the stability of the future electricity supply requires the design and development of new rapid protection schemes.

\section{New electricity producers}

The rise of renewables has added many new energy producers, because each wind turbine or solar panel is a power plant in its own right. Suddenly, citizens with their own solar panels are prosumers, producers and consumers at the same time. As a supplier, they are highly variable, as wind speeds and solar radiation fluctuate. As a result, the current in the power grid can flow in two directions and change its direction quickly. The big change for the whole system is that the distribution part of the electricity supply, which until now was passive - consumption of electricity - becomes an active part of the system - consumption and production of electricity.

\section{Electrification of society}

We also see a further electrification of society. Transport is becoming electric, as is heating, and industrial processes are largely driven by electricity. This not only means more and more electrical devices in the system, but also an increasing demand for sustainable electricity. In addition to electrification, digitalisation is a growing trend in society. The digitalisation of the electricity supply leads to more sensors in the system which deliver information about the status of the power supply and the condition of the components. Many new components, such as inverters, digital transformers and batteries, have built-in electronics, making them intelligent and enabling them to communicate with each other. The data are collected and processed by grid operators enabling them to take operational decisions. It leads to a smart grid system that provides better and automated control of the system. Connecting and integrating a broad variety of new components such as inverters and electric cars, and energy sources such as solar panels and wind farms to the grid, and merging and transporting electricity has become a complex challenge.

\section{Reliable power supply}

The most important condition that the transition of the power supply must meet is to maintain the high reliability of the current systems. The transition is often compared to renovating a store while the sale goes on. Less $\mathrm{CO}_{2}$ emission and more sustainability in the electricity $\bullet \bullet \bullet$

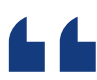
The inertia of the spinning rotor of an electrical generator is in stark contrast to the rapid response of solar panels to generate electricity. $"$ प 
- - sector requires radical actions, but we also have to ensure that everyone continues to receive electricity, heat and gas properly.

\section{The need for research facilities}

The growing share of renewable energy sources and power electronics components requires a new design and control of electricity grids. It also requires the introduction of new economic and financial models to give all stakeholders, including the prosumers, access to the energy market. Worldwide, universities are studying the different aspects of the energy transition to develop solutions to the challenges. Some are developing methodologies for planning, designing and operating smart medium and low-voltage networks with large-scale electricity generation from renewable energy sources and storage to increase flexibility of supply. Others focus on the theory of energy conversion and technologies for electromechanical energy conversion and power electronics. Modelling and simulations provide insights into topics such as supply and demand management, microgrids, integration of energy storage, markets and pricing mechanisms, and safety and security.

Today, most new consumer equipment, such as electric cars, batteries, mobile phones, computers or LED lamps, operate on DC power. But, as mentioned above, our electricity grid runs on alternating current, so inverters are needed to first convert DC electricity to $\mathrm{AC}$ and then vice versa. To prevent conversion losses, autonomous electricity networks, so-called microgrids, that only work with DC electricity are designed and tested. Inverters for the (contactless) charging of electric cars and bicycles are being designed. Scientists study the role of car batteries and neighbourhood batteries as a stabiliser of microgrids and also of the entire electricity system with large-scale power plants that increase the flexibility of electricity supply. The main challenge is the integration of new technologies and components for electricity generation and storage, digital technologies for information and communication and models for markets and control in the existing electricity system. And the integration must be done without compromising system performance. Stability, protection and cyber-physical security issues of the future system are studied and control rooms of the future are being developed that can control the ever-faster dynamics in the entire electricity grid and prevent instability in the grid.

\section{The ESP Lab}

To facilitate the research for the energy transition and to physically bring together the relevant research, TU Delft has opened on 1 October 2021, a new dedicated laboratory, the Electrical Sustainable Power Lab (ESP Lab). In the ESP Lab, crucial elements from the electricity grid, such as high-voltage facilities, wind and solar energy, energy storage and distribution networks, are combined into one functioning whole. The laboratory has advanced research and testing facilities to design and fabricate new materials, components, technologies and systems for the future sustainable energy system, and to characterise, optimise and validate them. Examples include solar cells, electronic power converters, electrical machines, microgrids, smart grids, high voltage $\mathrm{AC}$ and $\mathrm{DC}$ components, monitoring and diagnostics of components and power grids. The ESP Lab is ideally suited for the development of individual components and technologies needed for the future energy system.

To provide usable and proven solutions for a safe and reliable energy supply in the future, one must not only test the individual parameters of these new technologies and components, but, more importantly, one must test their performance at the system level. The ESP Lab offers scientists and researchers exactly this unique opportunity. It provides the facilities and a platform for Energy Transition Solutions to collaborate on integrated system solutions for various areas of electricity processing, such as electricity generation, transmission, distribution and use. Supercomputers that can simulate the behaviour of large power grids play an important role within the ESP Lab. What is special is that you can connect real devices in the lab to those powerful computers. For example, if you want to connect an offshore wind farm or a large neighbourhood battery to a power grid and you want to know what the consequences are for the stability of the grid. Or how you should intervene to keep the grid working properly. These questions can be answered using a digital twin, a digital copy of the electricity grid under investigation. On a digital copy, these kinds of experiments and investigations are carried out without running the risk of breaking anything in the real power grid. Smart equipment will play an increasingly important role for a sustainable and safe electricity grid. The smart electronics can make the grid more efficient and reliable if this equipment is controlled by good control systems. These control systems are being developed and tested by researchers in the ESP Lab using supercomputers.

With the results of the research, the ESP Lab can advise grid operators, who can then better deal with changes aimed at the electricity grid of the future.

\section{About the author}

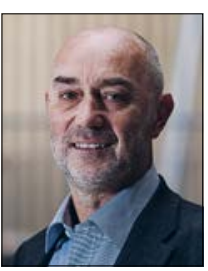

Miro Zeman is professor of Photovoltaic Technology at TU Delft. He chairs the Electrical Sustainable Energy department, which focuses on research into the sustainable and smart energy system of the future.M.Zeman@tudelft.nl 


\title{
Hitting New Milestones and Accelerating the Commercialisation of Quantum Computing in Europe
}

\author{
Just over a year ago Rigetti Computing, a California-based developer of quantum computers \\ was granted $£ 10$ Million by Innovate UK, the UK government's research innovation agency, \\ to accelerate the commercialisation of quantum computing in the UK.
}

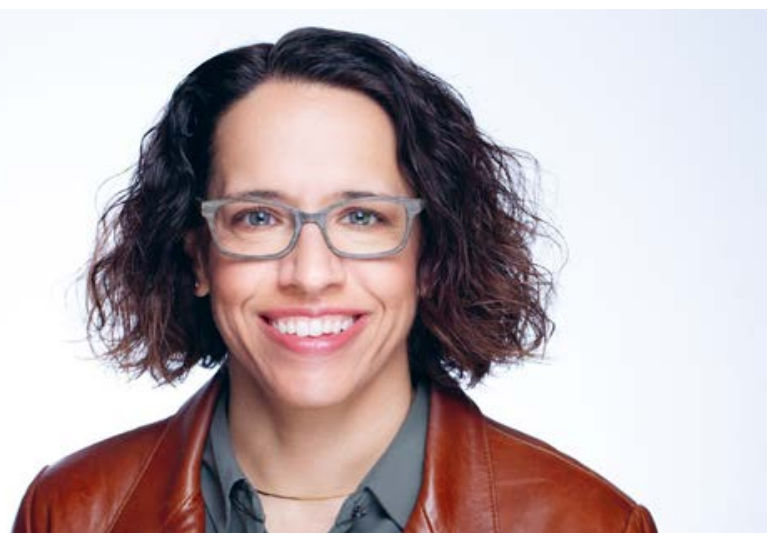

att Martin, Director of Engineering at Oxford Instruments NanoScience, talks to Mandy Birch, SVP of Technology Partnerships at Rigetti, about the progress that has been made on the project in the last year. Birch also covers the importance of collaboration on a project of this scale, and the future goals for this project.

Can you provide an update on the Innovate UK project and what's been achieved in the past 12 months?

It's been a busy year but through the collaboration between the various partners involved, we have managed to reach a significant milestone in the development of our quantum system - the complete setup of a Rigetti quantum computer in the UK moving on to testing, characterisation and selection of the Quantum Processing Unit (QPU). This milestone has only been possible thanks to the contributions of the team and the close collaboration with Oxford Instruments in particular who provided the ProteoxLX cooling system and the set-up of the computing facility infrastructure in Oxford.

Who are the various partners that you're working with as part of the project?

In addition to Oxford Instruments, we are also working with the University of Edinburgh as well as Phasecraft and Standard Chartered through cloud-based quantum computing access to assist with development activities. While developing our system, the University of Edinburgh has supported foundational quantum machine learning (QML), while our partners at Phasecraft have been able to use quantum-mechanical simulations to develop higher-performance batteries. Meanwhile, our partnership with Standard Chartered allowed us to explore volatility predictions within the financial markets. It's exciting to consider just how many applications quantum computers can have.
What can we expect to see from this project in the future? We are currently targeting commercial availability in early 2022. But the work is far from over. We made sure to design our computer with future-proofing in mind. Using Ol's ProteoxLX system with its fully customisable, modular and removable secondary insert, this allows the qubit integration to be easily scalable to larger numbers of qubits and more advanced architectures, allows the resulting quantum computer to remain up to date as new processors become available.

Our goal for the future is to have the ability to solve realworld problems with the quantum computer within the next five years. In order to achieve this, we will focus on scaling the current application prototypes to larger qubit counts while mitigating computational errors, with the goal of upgrading the system and deploying a larger, higher quality QPU in the second half of 2022.

FOR MORE INFORMATION: https://nanoscience.oxinst.com/ blog/accelerating-uk-quantum-computing-with-rigetti.

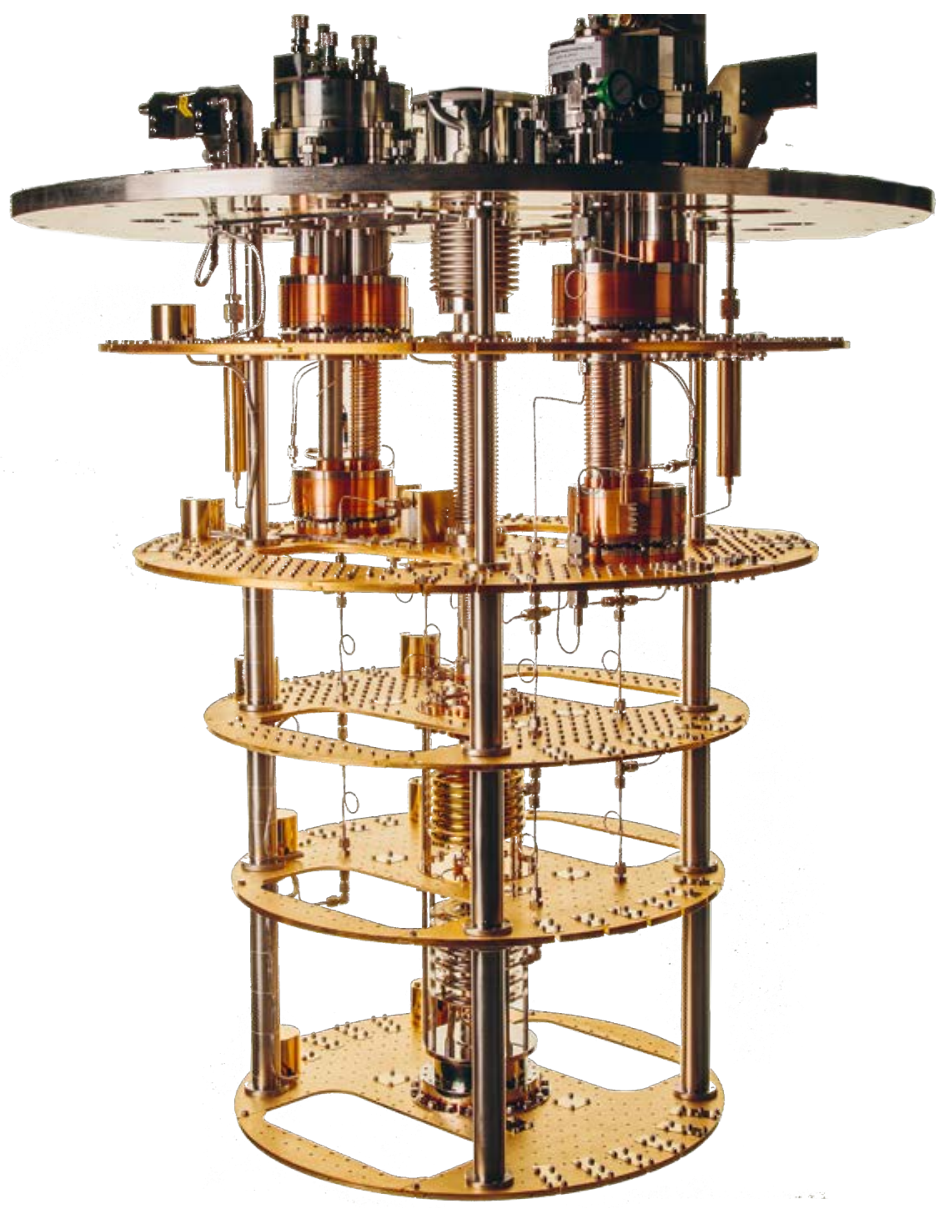

